\title{
Automated High-Quality TEM Sample Preparation
}

Yoh Yamamoto, Tatsuya Asahata, Masahiro Kiyohara, Hidekazu Suzuki, Haruo Takahashi, Yutaka Ikku, Makoto Sato, Hiroshi Matsumura, Kouji Iwasaki and Toshiaki Fujii

*SII NanoTechnology Inc., 36-1 Takenoshita Oyama-cho Sunto-gun Shizuoka, 410-1393,JAPAN

FIB is very attracvive technology for preparing lamellas for TEM observation due to its reliable imaging / processing-capability with nano-metric precision. Automation of TEM sample preparation using Ga FIB is important technology to improve throughput and reliability in fabrication of TEM samples. Currently with shrinkage of the size and new materials of semiconductor devise, requirements for high-quality sample preparation, i.e. preparation of thinner samples without FIB-induced damage is becoming more severe. Target thickness is required to be reduced down to $50 \mathrm{~nm}$. To meet this requirement, control of sample thickness and elimination of damage are important.

This report shows the automatic high-quality TEM samples pareparation with thickness monitoring function and low-accerelation voltage processing function.

Fig. 1 shows the flowchart of automatic high-qauality TEM sample preparation. The fine milling step has been devided into three steps in the newly developed technique.

The first step is the conventional thinning process with $30 \mathrm{kV}-\mathrm{Ga}$ ion.

The second step is the control of the sample thickness by in-situ SEM monitoring during FIB process. We established the sample thickness monitoring method by detecting backscattered electron generated in a thin sample. Irradiating a sample surface with electron beam (EB), secondary electron (SE) and backscattered electron (BSE) can be detected. Figure 2 shows the comparison between SE and BSE signal detected on the Si lamella. SE signal increases nonlinearly due to background SE generated by transmitted electrons behind the lamella(dotted circle in Fig.2). Because the detected SE signal includes the information of not only the surface of the lamella but also the background of the lamella, it is difficult to recognize thickness of the thin sample. On the other hand, most of the detected BSE is scattered in the shallow region from the sample surface; therefore, the information of the lamella itself can be detected. As shown in Fig.2, BSE signal intensity decreased in proportion to the sample thickness after the sample thickness becomes less than 200nm (dotted square in Fig.2). The point where BSE signal intensity started to decrease is determined by the relationship between material of the sample and energy of irradiated EB. Therefore, The sample thickness can be controlled by monitoring the decreased amount of BSE signal as shown in Fig.3. This thickness accuracy of this method was estimated less than $10 \mathrm{~nm}$ from Fig.3. With this method, the controllability of TEM sample thickness was improved.

The third step is fine milling to reduce damaged layer thickness. Processing with low acceleration voltage is accomplished with low-damaged sample preparation. With decreacing the acceleration voltage of FIB, FIB indused damage is reduced. On our Ga-FIB column, a stable and practical beam even at $1 \mathrm{kV}$ can be generated. Beam shift by changing the acceleration voltage can be compensate with drift correction function. This One-Point Drift Correction refers the image of the hole mark (one point) during FIB processing, and calibrates drift amount from a center of gravity of the image. By the calibration, it is possible to compensate the position displacement of beam irradiation precisely without influence of beam shape. The low acceleration votage processing as the final sequence of automatic TEM sample preparation enables to prepare low damaged sample.

As mentioned above, automated TEM sample preparation with thickness monitoring function and 
low-accerelation voltage process function enables automatic preparation of high-quality TEM samples with high reliability and high throughput.

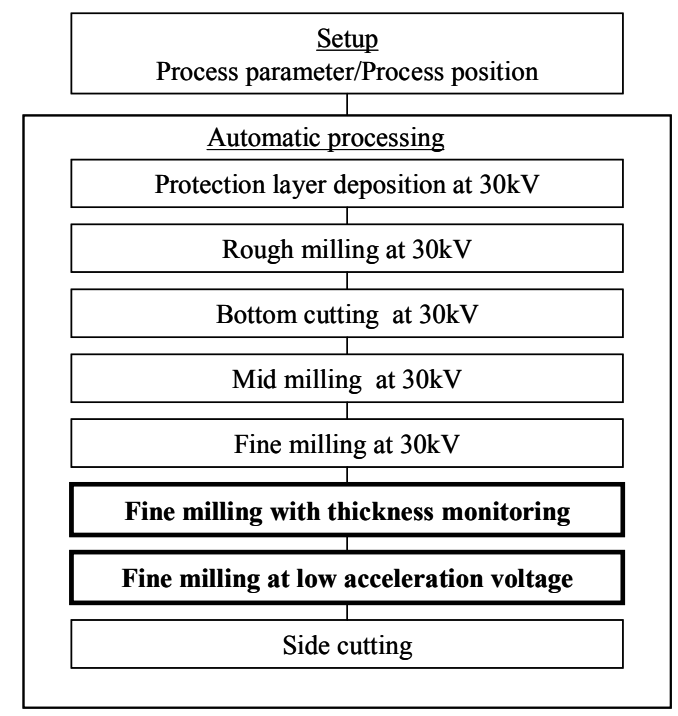

Fig. 1 Flowchart of TEM sample preparation Bold character: Added step

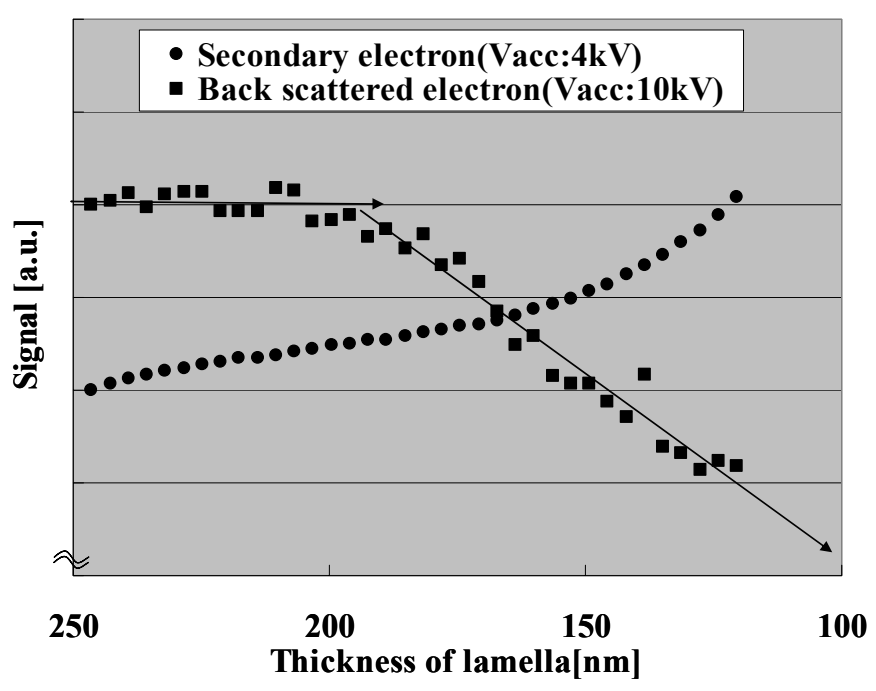

Fig. 2 Comparizon between SE and BSE signal

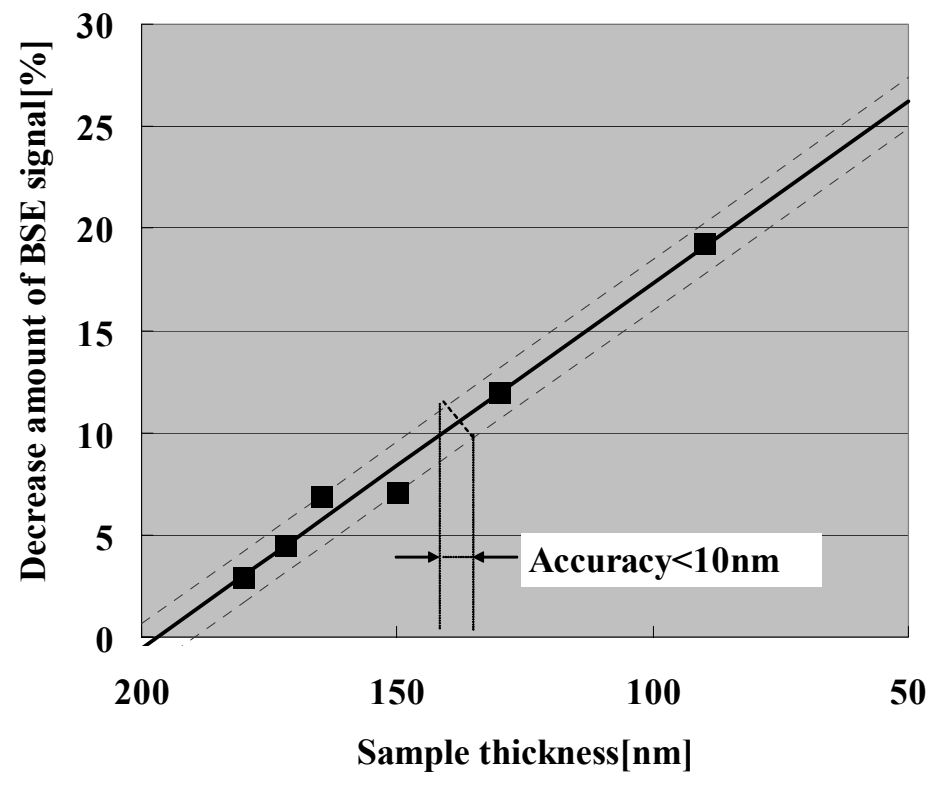

Fig. 3 Thickness monitoring with BSE signal 